\title{
High-order nonlinear optical response of a twisted bilayer graphene
}

\author{
Tatsuhiko N. Ikeda $\mathbb{1}$ \\ Institute for Solid State Physics, University of Tokyo, Kashiwa, Chiba 277-8581, Japan
}

(Received 8 May 2020; revised 27 June 2020; accepted 30 June 2020; published 14 July 2020)

\begin{abstract}
Focusing on the twist angle for the minimal commensurate structure, we perform nonperturbative calculations of electron dynamics in the twisted bilayer graphene (TBG) under intense laser fields. We show that the TBG exhibits enriched high-harmonic generation that cannot occur in monolayer or conventional bilayers. We elucidate the mechanism of these nonlinear responses by analyzing dynamical symmetries, momentum-resolved dynamics, and roles of interlayer coupling. Our results imply nonlinear "optotwistronics," or controlling optical properties of layered materials by artificial twists.
\end{abstract}

DOI: 10.1103/PhysRevResearch.2.032015

Introduction. Nonlinear optical response of materials [1,2] in intense optical fields have attracted growing attention since the invention of laser $[3,4]$. The high-harmonic generation in solids [5,6] is the prototypical nonlinear phenomenon [see Fig. 1(a)] and has seen a remarkable development in the last decade [7-11]. This phenomenon has attracted interest not only for compact frequency converter applications [12,13] but also as a probe of electron dynamics in intense optical fields [14]. Among various systems such as semiconductors [15-25], superconductors [26-28], strongly correlated systems [29-34], quantum magnets [35-37], and topological insulators [38,39], Dirac materials have turned out to have extremely large nonlinear susceptibility from the mid-infrared [40-42] down to the teraherz [43-45] frequency regimes. In particular, nonlinear response of graphene has been studied extensively [46-50].

Very recently, the twisted bilayer graphene (TBG) has opened a new avenue in physics of Dirac electrons in condensed matter [51,52]. The TBG consists of two sheets of graphene vertically stacked with an artificial twist angle, which enables us to manipulate electronic properties of layered materials [53] as sometimes called "twistronics" [54]. The twist angle brings about physical phenomena not present in a monolayer graphene such as superconductivity [51,55-57], Mott-like insulating states [51], to name a few. Microscopic theories [58-62] of the TBG have developed, and many active studies are going on to discover and understand novel phenomena $[63,64]$.

However, the nonlinear optical response of the TBG, or nonlinear "optotwistronics," has not yet been explored well. One theoretical challenge is that numerous electronic bands are involved in the TBG due to the large unit cell of the moiré structure. Recently, the Floquet band engineering has

Published by the American Physical Society under the terms of the Creative Commons Attribution 4.0 International license. Further distribution of this work must maintain attribution to the author(s) and the published article's title, journal citation, and DOI. been proposed based on the tight-binding model [66] and the low-energy effective Hamiltonian involving a few bands [67-69]. Another approach is the perturbation theory for the optical field. In this approach, the circular photogalvanic effect [70,71], one of the lowest-order nonlinear effects, has been found, but analyzing higher-order effects would become more challenging.

In this Rapid Communication, by restricting ourselves to a twist angle resulting in the minimal number of bands, we show that the TBG exhibits higher-order nonlinear responses that cannot happen in monolayer or conventional AA- or ABstacked bilayers. The restriction enables the nonperturbative calculation of electron dynamics in the full number of bands. We explain the nonlinear responses characteristic to the TBG by the dynamical symmetries of the Hamiltonian, where the key is that the TBG has a smaller point-group symmetry than monolayer or conventional bilayers. Thus the qualitative results shown in this work also apply to most twist angles leading to the same point-group symmetry. We also elucidate the mechanism of the nonlinear responses of the TBG by the reciprocal-space-resolved analysis and the decomposition of the electric current into the intralayer and interlayer contributions.

Model and setup. We begin by defining the lattice structure of the TBG that we study in this work. We consider two graphenes, or honeycomb lattices, on top of each other, i.e., the AA-stacked bilayer. We let $\boldsymbol{r}_{i}^{(l)}$ denote each site, where $l$ (= up or low) labels each layer and $i$ does each site within the layer. Thus $\boldsymbol{r}_{i}^{\text {(up) }}$ and $\boldsymbol{r}_{i}^{\text {(low) }}$ share their $x$ and $y$ components, but differ in their $z$ components: $\left[r_{i}^{(\text {up })}\right]_{z}-\left[r_{i}^{(\text {low })}\right]_{z}=d_{0}(\forall i)$ with $d_{0}$ being the interlayer distance.

The minimal commensurate TBG is obtained by rotating the upper (lower) layer by an angle $-\theta / 2(\theta / 2)$ with $\theta=21.79^{\circ}$ about the $z$-axis as illustrated in Fig. 1(b). Thus each site of the TBG is located at $\boldsymbol{R}_{i}^{\text {(up) }}=\mathcal{R}_{z}(-\theta / 2) \boldsymbol{r}_{i}^{\text {(up) }}$ and $\boldsymbol{R}_{i}^{\text {(low) }}=\mathcal{R}_{z}(\theta / 2) \boldsymbol{r}_{i}^{\text {(low) }}$, where $\mathcal{R}_{z}(\varphi)$ represents the $3 \times 3$ rotation matrix about the $z$ axis by angle $\varphi$. Here the commensurability means the presence of the exact discrete translation symmetry, and the unit cell contains 28 sites for $\theta=$ $21.79^{\circ}$. For other twist angles, the TBG has incommensurate 
(a)

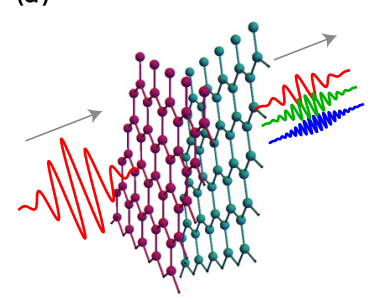

(c)

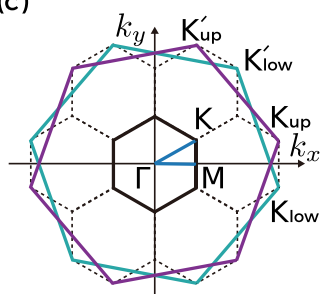

(d)

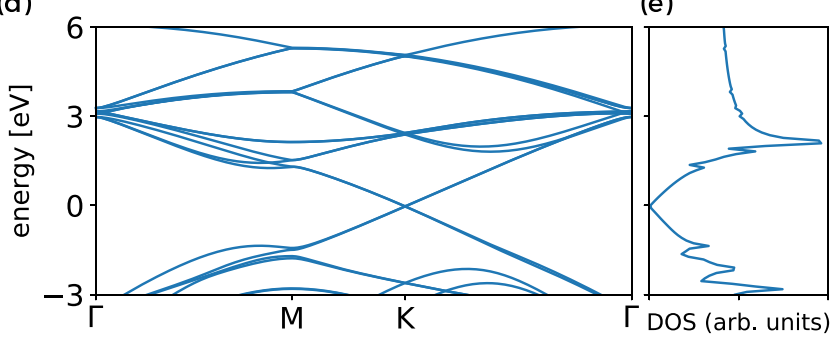

(b)

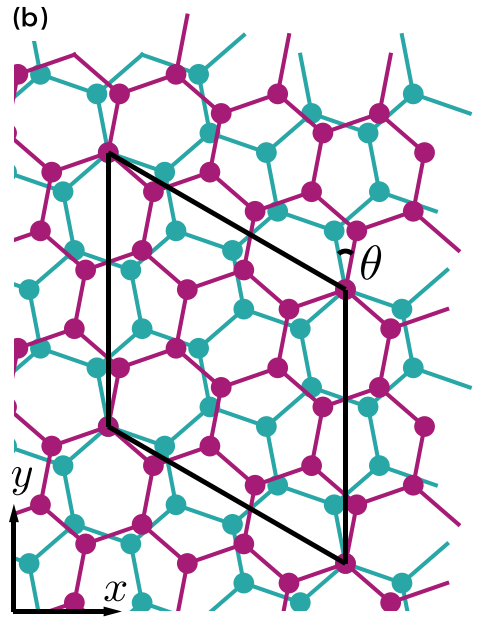

(e)

$\Gamma$ DOS (arb. units)

FIG. 1. (a) Schematic illustration of high-harmonic generation in twisted bilayer graphene. (b) Top view of the lattice structure of our TBG. The upper and lower layers rotate respectively by the angles $-\theta / 2$ and $\theta / 2$ with $\theta=21.79^{\circ}$ around a common A site. The parallelogram shows the unit cell involving 28 sites. (c) The central solid hexagon shows the first Brillouin zone (BZ) for the superlattice, and the dotted ones the other BZs. The larger hexagons represent the BZs for the upper and lower graphenes. (d) Electronic band structure around the Fermi energy (set to zero) together with (e) the corresponding density of states $[60,65]$.

structures or commensurate ones with larger unit cells. One exception is the $60^{\circ}$ twist, which gives the conventional ABstacked bilayer. However, as we will see below, the nonlinear optical responses for this case are similar to the monolayer or the AA-stacked bilayer.

To describe the quantum states of the electrons on the TBG, we adopt the tight-binding model of Refs. [60,72]

$$
H_{\mathrm{TBG}}=-\sum_{(i, l),\left(i^{\prime}, l^{\prime}\right)} t\left(\boldsymbol{R}_{i}^{(l)}, \boldsymbol{R}_{i^{\prime}}^{\left(l^{\prime}\right)}\right)\left|\boldsymbol{R}_{i}^{(l)}\right\rangle\left\langle\boldsymbol{R}_{i^{\prime}}^{\left(l^{\prime}\right)}\right|+\text { H.c. },
$$

where $\left|\boldsymbol{R}_{i}^{(l)}\right\rangle$ denotes the Wannier state at position $\boldsymbol{R}_{i}^{(l)}$. The transfer integral $t\left(\boldsymbol{R}_{i}^{(l)}, \boldsymbol{R}_{i^{\prime}}^{\left(l^{\prime}\right)}\right)$ between $\boldsymbol{R}_{i}^{(l)}$ and $\boldsymbol{R}_{i^{\prime}}^{\left(l^{\prime}\right)}$ depends only on the distance $\left|\boldsymbol{R}_{i}^{(l)}-\boldsymbol{R}_{i^{\prime}}^{\left(l^{\prime}\right)}\right|$ and its parametrization is taken from Refs. [60,72]. By the Fourier transform in the $x y$ plane, we obtain the reciprocal-lattice representation: $H_{\mathrm{TBG}}=\sum_{\boldsymbol{k}, \mu, l, v, l^{\prime}} h_{\mu l, v l^{\prime}}(\boldsymbol{k})|\boldsymbol{k} ; \mu, l\rangle\left\langle\boldsymbol{k} ; \nu, l^{\prime}\right|$, where $\boldsymbol{k}=$ $\left(k_{x}, k_{y}\right)$ is the two-dimensional wave vector and the pair $(\mu, l)$ ( $\mu=1,2, \ldots, 14$ and $l=$ up or low) serves as the internal degree of freedom corresponding to each site in the TBG unit cell.

The band structure of our TBG is obtained from the eigenvalues of the $28 \times 28$ Hamiltonian matrix $h_{\mu l, v l^{\prime}}(\boldsymbol{k})$ and

shown in Fig. 1(d) (see also Ref. [60]). Throughout this work, we assume the half-filling and set $E_{F}=0$. We remark that the Dirac cone at the $K$ point is approximately doubly degenerate besides the spin degeneracy. This degeneracy comes from the Dirac electrons of the upper and lower layers. The interlayer coupling does not affect these Dirac electrons much but causes band splittings away from the $K$ point.

Now we introduce the coupling of the TBG to the laser propagating in the $z$ direction. Considering that the laser wavelength is larger enough than the interatomic distances, we assume that the laser electric field $\boldsymbol{E}(t)=\left(E_{x}(t), E_{y}(t), 0\right)$ is homogeneous. Then the coupling energy is given by

$$
H_{\mathrm{ext}}(t)=\sum_{(i, l)} e \boldsymbol{E}(t) \cdot \boldsymbol{R}_{i}^{(l)}\left|\boldsymbol{R}_{i}^{(l)}\right\rangle\left\langle\boldsymbol{R}_{i}^{(l)}\right|,
$$

where $e$ is the elementary charge. The total Hamiltonian in the Fourier representation is given by $\hat{H}_{\text {total }}(t) \equiv H_{\mathrm{TBG}}+$ $H_{\mathrm{ext}}(t)=\sum_{\boldsymbol{k}, \mu, l, v, l^{\prime}} h_{\mu l, v l^{\prime}}(\boldsymbol{k}+e \boldsymbol{A}(t))|\boldsymbol{k} ; \mu, l\rangle\left\langle\boldsymbol{k} ; v, l^{\prime}\right|$,

where the vector potential $\boldsymbol{A}(t)=-\int^{t} \boldsymbol{E}_{2 d}\left(t^{\prime}\right) d t^{\prime}$ with $\boldsymbol{E}_{2 d}(t)=\left(E_{x}(t), E_{y}(t)\right)$.

We focus on a pulse laser of angular frequency $\Omega$,

$$
\boldsymbol{A}(t)=\frac{E_{0}}{\Omega} f(t)\left[\begin{array}{c}
\cos (\Omega t) \\
\epsilon_{p} \sin (\Omega t)
\end{array}\right],
$$

where $f(t)$ represents a five-cycle Gaussian envelope function [73] and $E_{0}$ approximately gives the peak electric-field amplitude. We set the angular frequency as $\hbar \Omega=0.3 \mathrm{eV}$ corresponding to a mid-infrared laser widely used in experiments (see, e.g., Refs. [5,40]). The parameter $\epsilon_{p}$ distinguishes the polarization: $\epsilon_{p}=0$ means the linear polarization along the $x$ direction and $\epsilon_{p}=1$ the circular polarization.

Our simulation protocol is as follows. At the initial time $t=t_{\text {ini }}(\ll 0)$, we take the ground state in which every energy eigenstate with negative (positive) energy is occupied (unoccupied). Since we neglect interactions between electrons, we numerically solve the time-dependent Schrödinger equation for individual occupied state under $\hat{H}_{\text {total }}(t)$. To reduce the computational cost, we ignore the time evolution of occupied states well below the Fermi energy $\left(E<E_{F}-5 \hbar \Omega\right)$ since their contributions to the electric current are small. To analyze the optical response, we consider the electric current

$$
\hat{\boldsymbol{J}}(t)=\frac{\partial \hat{H}_{\text {total }}(t)}{\partial \boldsymbol{A}(t)}=\sum_{\boldsymbol{k}} \hat{\boldsymbol{J}}(\boldsymbol{k} ; t)
$$

and its expectation value $\boldsymbol{J}(t)=\sum_{\boldsymbol{k}} \boldsymbol{J}(\boldsymbol{k} ; t)=\sum_{\boldsymbol{k}}\langle\hat{\boldsymbol{J}}(\boldsymbol{k} ; t)\rangle_{t}$ at each time step. Further technical details are described in Ref. [73].

High-harmonic generation. First, we analyze the spectra for the electric current induced by the linearly polarized laser. Figures 2(a) and 2(b) show the spectra of the currents parallel $\left(J_{x}\right)$ and perpendicular $\left(J_{y}\right)$ to the electric field, respectively. We observe several peaks at $(2 m+1) \Omega$ for $J_{x}$ and at $2 m \Omega$ for $J_{y}(m \in \mathbb{Z})$. In experiments, the induced current with these harmonic peaks is observed as the high-harmonic generation from the TBG as illustrated in Fig. 1(a).

The even-order harmonics are characteristic to the TBG and cannot appear in the monolayer or conventional AAand AB-stacked bilayers [74] that have inversion centers [75] although the interlayer bias can give rise to the evenorder harmonics $[76,77]$. The selection rules that $J_{x}\left(J_{y}\right)$ has 
(a)

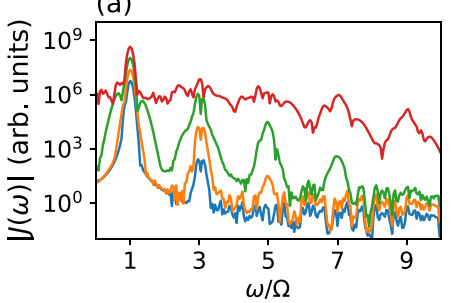

(c)

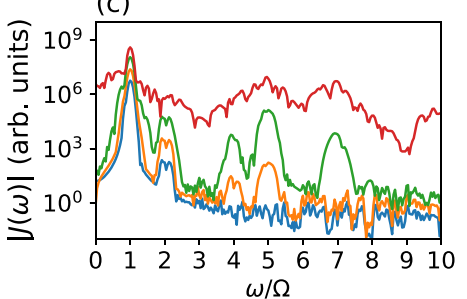

(e)
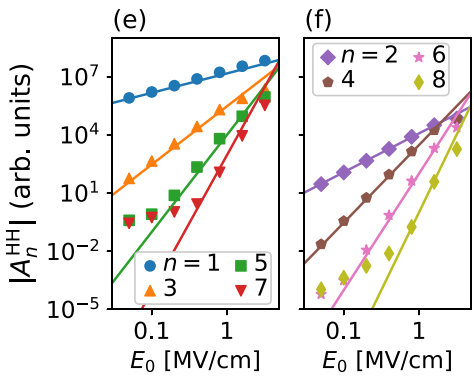

(g)
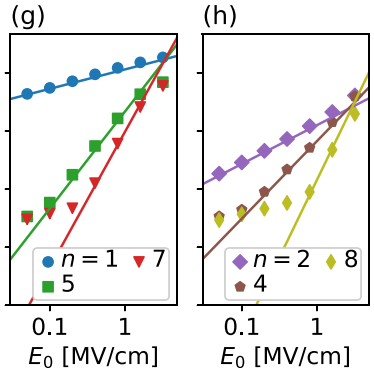

FIG. 2. [(a)-(d)] Amplitude spectra for in-plane components of electric current plotted for input electric fields $E_{0}=0.05$ (blue), 0.2 (orange), 0.8 (green), and $1.6 \mathrm{MV} / \mathrm{cm}$ (red). The polarization of the input electric field is linear (along $x$ ) for (a) and (b) and circular for (c) and (d), and the electric-current component is $J_{x}$ for (a) and (c) and $J_{y}$ for (b) and (d). [(e)-(h)] Amplitudes of $n$th harmonic $A_{n}^{\mathrm{HH}}$ plotted against the input field amplitude $E_{0}$. In (e) and (h), the nonvanishing harmonics of $J_{x}$ and $J_{y}$ for linearly polarized fields are plotted, respectively. In (g) and (h), we plot the nonvanishing harmonics of $J_{x}$ in the circularly polarized fields at the odd and even orders respectively. In (e)-(h), the solids lines show the eye guides $\propto E_{0}^{n}$ for each $n$.

odd-only (even-only) harmonics are explained by the socalled dynamical symmetry appearing in the limit of $t_{\mathrm{FWHM}} \rightarrow$ $\infty[78,79]$. Note that our TBG without the laser field has the symmetry under $C_{2 y}$, i.e., the $\pi$ rotation about the $y$ axis [see Fig. 1(b)]. In the presence of the linearly polarized electric field, this symmetry is no longer true, but $C_{2 y}$ combined with the time translation $t \rightarrow t+T / 2$ becomes a symmetry transformation. This dynamical symmetry leads to the selection rules together with the fact that $J_{x}\left(J_{y}\right)$ is odd (even) under the transformation (see Ref. [73] for details).

To analyze the amplitude of the $n$th harmonic, we define the following quantity: $A_{n}^{\mathrm{HH}} \equiv \int_{(n-1 / 2) \Omega}^{(n+1 / 2) \Omega} \frac{d \omega}{\Omega} J(\omega)$, where $J(\omega)$ represents the spectrum of some component of electric current. In Figs. 2(e) and 2(f), we plot the harmonic amplitude for $n \leqslant 8$ against the incident field amplitude $E_{0}$. For $E_{0} \leqslant$ $1 \mathrm{MV} / \mathrm{cm}$, each harmonic amplitude scales as $A_{n}^{\mathrm{HH}} \propto E_{0}^{n}$ in line with the perturbation theory [2]. On the other hand, in the strong-field regime $E_{0} \geqslant 1 \mathrm{MV} / \mathrm{cm}, A_{n}^{\mathrm{HH}}$ slightly saturates and deviates from the $E_{0}^{n}$ scaling. In this regime, the harmonic (a)

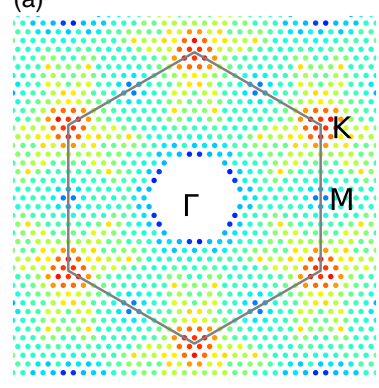

(b)

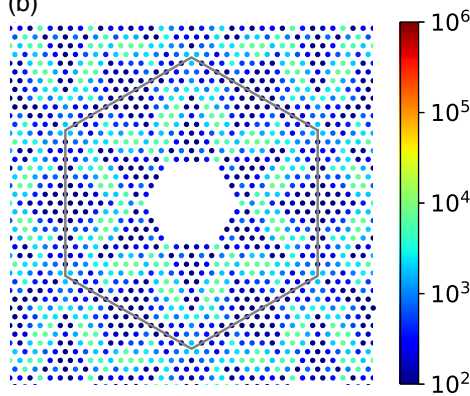

FIG. 3. (a) $\boldsymbol{k}$-resolved harmonic amplitude $\left|A_{n}^{\mathrm{HH}}(\boldsymbol{k})\right|$ for $n=2$ over the $\boldsymbol{k}$ space. (b) Absolute value of the sixfold-rotation sum $S_{n}^{\mathrm{HH}}(\boldsymbol{k})$ for $n=2$ (see text for definition). In both panels, we use the extended zone scheme, duplicating the data outside the first BZ.

peaks are not very sharp as shown in Figs. 1(a) and 1(b) due to lots of excitations occurring between the bands.

Second, we analyze the case of the circular polarization. The current spectra for $J_{x}$ and $J_{y}$ are shown in Figs. 2(c) and $2(\mathrm{~d})$, in which we find a peculiar selection rule: The harmonics at $3 m \Omega(m \in \mathbb{Z})$ are prohibited. This selection rule derives from another dynamical symmetry consisting of $C_{3}$, the $120^{\circ}$ rotation about the $z$ axis, and the time translation $t \rightarrow$ $t+T / 3$. This dynamical symmetry allows the harmonics only at $(3 m \pm 1) \Omega$ and hence prohibits $3 m \Omega$. This symmetry argument also implies that $\boldsymbol{J}(\omega=3 m \pm 1)$ are circularly polarized $[73,79]$, and thus we obtain similar harmonic peak heights for $J_{x}$ and $J_{y}$ in Figs. 2(c) and 2(d). The harmonic amplitudes and their saturation behavior are shown in Figs. 2(g) and 2(h).

The peculiar selection rule under the circularly polarized field is characteristic of the TBG and not present in the monolayer or conventional bilayers. The monolayer and the AA-stacked bilayer have the sixfold rotational symmetry, and thus the harmonics are allowed only for $(6 m \pm 1) \Omega$ [78]. The AB-stacked bilayer also allows only harmonics at $(6 m \pm 1) \Omega$ due to the threefold-rotation and inversion symmetries. These symmetries forbid the harmonics $3 m \Omega$ and $2 m \Omega$, respectively, and the allowed harmonics are only $(6 m \pm 1) \Omega$. The TBG is less symmetric than the monolayer and conventional bilayers, exhibiting enriched nonlinear optical responses with orders $n=6 m \pm 2$.

Reciprocal-space analysis. Having found the harmonic responses characteristic to the TBG, we now investigate their mechanism. To this end, we look into the harmonic amplitude resolved in the reciprocal space by introducing $A_{n}^{\mathrm{HH}}(\boldsymbol{k}) \equiv$ $\int_{(n-1 / 2) \Omega}^{(n+1 / 2) \Omega} \frac{d \omega}{\Omega} J(\boldsymbol{k} ; \omega)$, where $J(\boldsymbol{k} ; \omega)$ represents some component of the Fourier transform of $\boldsymbol{J}(\boldsymbol{k} ; t)$.

Figure 3(a) shows the $\boldsymbol{k}$-resolved second harmonic amplitude $\left|A_{n=2}^{\mathrm{HH}}(\boldsymbol{k})\right|$ obtained for the circularly polarized field with $E_{0}=0.8 \mathrm{MV} / \mathrm{cm}$. The largest amplitude exists in the vicinity of the $K$ and $K^{\prime}$ points and this tendency is commonly seen for the other harmonic orders $n$. This observation means that large nonlinear currents are carried by the Dirac electrons [see Fig. 1(d)] consistently with the experimental results showing that the Dirac electrons generate harmonics very efficiently $[44,45]$.

Nevertheless, nonDirac electrons play more significant roles in the second harmonic after the sum over 

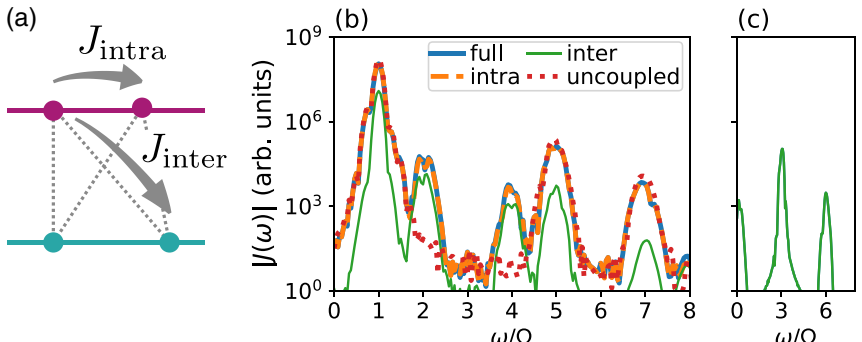

FIG. 4. (a) Schematic illustration of intralayer and interlayer electric currents in the side view of the TBG. [(b) and (c)] Amplitude spectra of the (b) in-plane $\left(J_{x}\right)$ and (b) out-of-plane $\left(J_{z}\right)$ electric current generated by the circular polarization with $E_{0}=0.8 \mathrm{MV} / \mathrm{cm}$. Each spectrum represents the total $\boldsymbol{J}$, intralayer $\boldsymbol{J}_{\text {intra }}$, and interlayer $\boldsymbol{J}_{\text {inter }}$ currents in the TBG and the total current in the uncoupled bilayers.

the BZ. To show this, we focus on the sixfold-rotation sum of the $\boldsymbol{k}$-resolved harmonics and define $S_{n}^{\mathrm{HH}}(\boldsymbol{k}) \equiv$ $\sum_{\ell=0}^{5} A_{n}^{\mathrm{HH}}\left(\mathcal{R}_{z}(\pi / 3)^{\ell} \boldsymbol{k}\right)$. We note that the total harmonic amplitude $A_{n}^{\mathrm{HH}}$ is obtained as a weighted sum of $S_{n}^{\mathrm{HH}}(\boldsymbol{k})$. Figure 3(b) shows $\left|S_{n=2}^{\mathrm{HH}}(\boldsymbol{k})\right|$ over the $\boldsymbol{k}$-space, in which we find that the $\boldsymbol{k}$ points near the $K$ point give small contributions. Indeed the individual Dirac electrons carry large nonlinear currents, but these currents cancel each other very strongly. As a result, the nonDirac electrons in the middle of the $\mathrm{BZ}$ give more contributions for the second harmonic. The importance of nonDirac electrons are common with other harmonic orders $n=6 m \pm 2$ that are characteristic to the TBG, whereas the Dirac electrons give dominant contributions for the ordinary harmonics $n=6 m \pm 1$.

The band structure in Fig. 1(d) confirms this interpretation. As noted above, the interlayer coupling, emerging as small band splittings, is more effective away from the $K$ point. Given that the interlayer coupling activates the characteristic harmonics $n=6 m \pm 2$, they are contributed from the $\boldsymbol{k}$ points away from the $K$ point.

Role of interlayer coupling. To elucidate other aspects of the interlayer coupling, we decompose the total electric current into two parts, the intralayer and interlayer contributions, as

$$
\boldsymbol{J}(t)=\boldsymbol{J}_{\text {intra }}(t)+\boldsymbol{J}_{\text {inter }}(t)
$$

The definitions of these contributions follow from the fact that the current operator $\hat{\boldsymbol{J}}(\boldsymbol{k} ; t)$ has a $28 \times 28$-matrix representation $\hat{\boldsymbol{J}}(\boldsymbol{k} ; t)=\sum_{\mu, l, v, l^{\prime}} j_{\mu l, v l^{\prime}}(\boldsymbol{k})|\boldsymbol{k} ; \mu, l\rangle\left\langle\boldsymbol{k} ; v, l^{\prime}\right|$. We define the operators $\hat{\boldsymbol{J}}_{\text {intra }}$ and $\hat{\boldsymbol{J}}_{\text {inter }}$ as the $l=l^{\prime}$ and $l \neq l^{\prime}$ parts of $\hat{\boldsymbol{J}}(\boldsymbol{k} ; t)$, respectively, and $\boldsymbol{J}_{\text {intra }}(t)$ and $\boldsymbol{J}_{\text {inter }}(t)$ are their expectation values. Figure 4(a) schematically illustrates $\hat{\boldsymbol{J}}_{\text {intra }}$ and $\hat{\boldsymbol{J}}_{\text {inter }}$, which are the electric currents accompanied by the intralayer and interlayer hoppings of electrons, respectively.

The intralayer component gives the dominant contribution as shown in Fig. 4(b), which shows the result for the circular polarization with $E_{0}=0.8 \mathrm{MV} / \mathrm{cm}$. Since the $x$ and $y$ components are essentially equivalent for the circular polarization, we plot only the $x$ component.

For comparison, we also plot the result for the uncoupled bilayers which are defined by removing all the interlayer hopping, i.e., setting $t\left(\boldsymbol{R}_{i}^{(l)}, \boldsymbol{R}_{i^{\prime}}^{\left(l^{\prime}\right)}\right)=0$ for $l \neq l^{\prime}$. Similarly to the monolayer, the uncoupled bilayers only give the harmonics at $n=6 m \pm 1$. For these harmonics, the difference between the TBG and uncoupled bilayers is quite small, meaning that they are carried by the electrons accelerated within each layer.

Remarkably, the dominance of the intralayer current holds also for the harmonics $n=6 m \pm 2$ that are caused by the interlayer coupling. Indeed the interlayer coupling is important and, as shown in Fig. 4(c), there occurs significant charge transfer between the layers including some dc $(0 \Omega)$ component corresponding to the photogalvanic effect $[70,71]$. However, the in-plane currents accompanied by the interlayer hopping give less contribution to the total current. Rather, the in-plane currents are contributed more by the intralayer electron hopping, and the interlayer coupling assists them by breaking higher symmetry of the uncoupled bilayers and preventing the harmonic currents from canceling out in the BZ.

Discussions and conclusions. We have conducted the nonperturbative calculations of the laser-induced electric currents in the minimal commensurate TBG, finding higher-order harmonic responses that are not present in monolayer or conventional bilayers. In contrast to the common harmonics, these new harmonics are carried more by nonDirac electrons and caused by the interplay between the intralayer and interlayer electron hoppings. The selection rules of the harmonics are qualitatively distinct and could be tested within the current optics technology. Since the point-group symmetry of the TBG is common for most twist angles, the selection rules found here should also apply to other twist angles. The enriched harmonics in the TBG offer versatile frequency-conversion channels for future applications.

An important future direction toward nonlinear "optotwistronics" is to unravel the dependence on the twist angle, which has been fixed to $\theta=21.79^{\circ}$ in this work. Qualitative results might be different for smaller angles and lowerfrequency lasers since there occur some emergent symmetries $[80,81]$. In addition, $\theta=30^{\circ}$ is a particularly important twist angle, at which the TBG becomes a quasicrystal and can accommodate symmetries prohibited in ordinary crystals [82-84]. Another direction is to go into the deep nonperturbative regime with even stronger fields. In this regime, one should include relaxation due to, e.g., the interband dephasing [85] and impurity scattering [86,87]. We leave these open issues for future study.

Note added. "Optotwistronics" discussed here is also called as "twistoptics" [88].

Acknowledgment. This work was supported by JSPS KAKENHI Grant No. JP18K13495.
[1] P. A. Franken, A. E. Hill, C. W. Peters, and G. Weinreich, Phys. Rev. Lett. 7, 118 (1961).
[2] R. W. Boyd, Nonlinear Optics, 3rd ed. (Academic Press, Orlando, FL, 2008). 
[3] A. L. Schawlow and C. H. Townes, Phys. Rev. 112, 1940 (1958).

[4] T. H. Maiman, Nature 187, 493 (1960).

[5] S. Ghimire, A. D. DiChiara, E. Sistrunk, P. Agostini, L. F. DiMauro, and D. A. Reis, Nat. Phys. 7, 138 (2011).

[6] O. Schubert, M. Hohenleutner, F. Langer, B. Urbanek, C. Lange, U. Huttner, D. Golde, T. Meier, M. Kira, S. W. Koch, and R. Huber, Nat. Photon. 8, 119 (2014).

[7] M. Hohenleutner, F. Langer, O. Schubert, M. Knorr, U. Huttner, S. W. Koch, M. Kira, and R. Huber, Nature 523, 572 (2015).

[8] G. Ndabashimiye, S. Ghimire, M. Wu, D. A. Browne, K. J. Schafer, M. B. Gaarde, and D. A. Reis, Nature (London) 534, 520 (2016).

[9] Y. S. You, D. Reis, and S. Ghimire, Nat. Phys. 13, 345 (2017).

[10] T. Higuchi, C. Heide, K. Ullmann, H. B. Weber, and P. Hommelhoff, Nature (London) 550, 224 (2017).

[11] K. Kaneshima, Y. Shinohara, K. Takeuchi, N. Ishii, K. Imasaka, T. Kaji, S. Ashihara, K. L. Ishikawa, and J. Itatani, Phys. Rev. Lett. 120, 243903 (2018).

[12] S. Ghimire, G. Ndabashimiye, A. D. DiChiara, E. Sistrunk, M. I. Stockman, P. Agostini, L. F. DiMauro, and D. A. Reis, J. Phys. B: At. Mol. Opt. Phys. 47, 204030 (2014).

[13] S. Ghimire and D. A. Reis, Nat. Phys. 15, 10 (2019).

[14] G. Vampa, T. J. Hammond, N. Thiré, B. E. Schmidt, F. Légaré, C. R. McDonald, T. Brabec, D. D. Klug, and P. B. Corkum, Phys. Rev. Lett. 115, 193603 (2015).

[15] D. Golde, T. Meier, and S. W. Koch, J. Opt. Soc. Am. B 23, 2559 (2006).

[16] D. Golde, T. Meier, and S. W. Koch, Phys. Rev. B 77, 075330 (2008).

[17] M. Wu, S. Ghimire, D. A. Reis, K. J. Schafer, and M. B. Gaarde, Phys. Rev. A 91, 043839 (2015).

[18] G. Vampa, C. R. McDonald, G. Orlando, P. B. Corkum, and T. Brabec, Phys. Rev. B 91, 064302 (2015).

[19] T. Ikemachi, Y. Shinohara, T. Sato, J. Yumoto, M. KuwataGonokami, and K. L. Ishikawa, Phys. Rev. A 95, 043416 (2017).

[20] E. N. Osika, A. Chacón, L. Ortmann, N. Suárez, J. A. Pérez-Hernández, B. Szafran, M. F. Ciappina, F. Sols, A. S. Landsman, and M. Lewenstein, Phys. Rev. X 7, 021017 (2017).

[21] T.-Y. Du and X.-B. Bian, Opt. Express 25, 151 (2017).

[22] F. Catoire, H. Bachau, Z. Wang, C. Blaga, P. Agostini, and L. F. DiMauro, Phys. Rev. Lett. 121, 143902 (2018).

[23] T. N. Ikeda, K. Chinzei, and H. Tsunetsugu, Phys. Rev. A 98 , 063426 (2018).

[24] F. Navarrete, M. F. Ciappina, and U. Thumm, Phys. Rev. A 100, 033405 (2019).

[25] P. Xia, T. Tamaya, C. Kim, F. Lu, T. Kanai, N. Ishii, J. Itatani, H. Akiyama, and T. Kato, arXiv:2004.04492.

[26] R. Matsunaga, N. Tsuji, H. Fujita, A. Sugioka, K. Makise, Y. Uzawa, H. Terai, Z. Wang, H. Aoki, and R. Shimano, Science 345, 1145 (2014).

[27] Y. Kawakami, T. Amano, Y. Yoneyama, Y. Akamine, H. Itoh, G. Kawaguchi, H. M. Yamamoto, H. Kishida, K. Itoh, T. Sasaki, S. Ishihara, Y. Tanaka, K. Yonemitsu, and S. Iwai, Nat. Photon. 12, 474 (2018).

[28] S. Nakamura, K. Katsumi, H. Terai, and R. Shimano, arXiv:2003.00417.
[29] T. Ikemachi, Y. Shinohara, T. Sato, J. Yumoto, M. KuwataGonokami, and K. L. Ishikawa, Phys. Rev. A 98, 023415 (2018).

[30] Y. Murakami, M. Eckstein, and P. Werner, Phys. Rev. Lett. 121, 057405 (2018).

[31] S. Imai, A. Ono, and S. Ishihara, Phys. Rev. Lett. 124, 157404 (2020).

[32] M. Lysne, Y. Murakami, and P. Werner, Phys. Rev. B 101, 195139 (2020).

[33] A. Roy, S. Bera, and K. Saha, arXiv:2003.10253.

[34] C.-M. Wang, N. Tancogne-Dejean, M. Altarelli, A. Rubio, and S. A. Sato, arXiv:2004.09049.

[35] J. Lu, X. Li, H. Y. Hwang, B. K. Ofori-Okai, T. Kurihara, T. Suemoto, and K. A. Nelson, Phys. Rev. Lett. 118, 207204 (2017).

[36] S. Takayoshi, Y. Murakami, and P. Werner, Phys. Rev. B 99, 184303 (2019).

[37] T. N. Ikeda and M. Sato, Phys. Rev. B 100, 214424 (2019).

[38] D. Bauer and K. K. Hansen, Phys. Rev. Lett. 120, 177401 (2018).

[39] C. Jürß and D. Bauer, Phys. Rev. B 99, 195428 (2019).

[40] N. Yoshikawa, T. Tamaya, and K. Tanaka, Science (NY) 356, 736 (2017).

[41] M. Baudisch, A. Marini, J. D. Cox, T. Zhu, F. Silva, S. Teichmann, M. Massicotte, F. Koppens, L. S. Levitov, F. J. García de Abajo, and J. Biegert, Nat. Commun. 9, 1018 (2018).

[42] T. Jiang, D. Huang, J. Cheng, X. Fan, Z. Zhang, Y. Shan, Y. Yi, Y. Dai, L. Shi, K. Liu, C. Zeng, J. Zi, J. E. Sipe, Y.-R. Shen, W.-T. Liu, and S. Wu, Nat. Photon. 12, 430 (2018).

[43] H. A. Hafez, S. Kovalev, J.-C. Deinert, Z. Mics, B. Green, N. Awari, M. Chen, S. Germanskiy, U. Lehnert, J. Teichert, Z. Wang, K.-J. Tielrooij, Z. Liu, Z. Chen, A. Narita, K. Müllen, M. Bonn, M. Gensch, and D. Turchinovich, Nature (London) 561, 507 (2018).

[44] B. Cheng, N. Kanda, T. N. Ikeda, T. Matsuda, P. Xia, T. Schumann, S. Stemmer, J. Itatani, N. P. Armitage, and R. Matsunaga, Phys. Rev. Lett. 124, 117402 (2020).

[45] S. Kovalev, R. M. A. Dantas, S. Germanskiy, J.-C. Deinert, B. Green, I. Ilyakov, N. Awari, M. Chen, M. Bawatna, J. Ling, F. Xiu, P. H. M. van Loosdrecht, P. Surówka, T. Oka, and Z. Wang, Nat. Commun. 11, 2451 (2020).

[46] S. A. Mikhailov, Europhys. Lett. 79, 27002 (2007).

[47] A. R. Wright, X. G. Xu, J. C. Cao, and C. Zhang, Appl. Phys. Lett. 95, 072101 (2009).

[48] K. L. Ishikawa, Phys. Rev. B 82, 201402(R) (2010).

[49] I. Al-Naib, J. E. Sipe, and M. M. Dignam, Phys. Rev. B 90, 245423 (2014).

[50] H. Rostami and V. Juričić, Phys. Rev. Res. 2, 13069 (2020).

[51] Y. Cao, V. Fatemi, S. Fang, K. Watanabe, T. Taniguchi, E. Kaxiras, and P. Jarillo-Herrero, Nature (London) 556, 43 (2018).

[52] Y. Cao, V. Fatemi, A. Demir, S. Fang, S. L. Tomarken, J. Y. Luo, J. D. Sanchez-Yamagishi, K. Watanabe, T. Taniguchi, E. Kaxiras, R. C. Ashoori, and P. Jarillo-Herrero, Nature (London) 556, 80 (2018).

[53] K. Tran, G. Moody, F. Wu, X. Lu, J. Choi, K. Kim, A. Rai, D. A. Sanchez, J. Quan, A. Singh, J. Embley, A. Zepeda, M. Campbell, T. Autry, T. Taniguchi, K. Watanabe, N. Lu, S. K. 
Banerjee, K. L. Silverman, S. Kim, E. Tutuc, L. Yang, A. H. MacDonald, and X. Li, Nature (London) 567, 71 (2019).

[54] S. Carr, D. Massatt, S. Fang, P. Cazeaux, M. Luskin, and E. Kaxiras, Phys. Rev. B 95, 075420 (2017).

[55] X. Lu, P. Stepanov, W. Yang, M. Xie, M. A. Aamir, I. Das, C. Urgell, K. Watanabe, T. Taniguchi, G. Zhang, A. Bachtold, A. H. MacDonald, and D. K. Efetov, Nature (London) 574, 653 (2019).

[56] M. Yankowitz, S. Chen, H. Polshyn, Y. Zhang, K. Watanabe, T. Taniguchi, D. Graf, A. F. Young, and C. R. Dean, Science 363, 1059 (2019).

[57] H. Isobe, N. F. Q. Yuan, and L. Fu, Phys. Rev. X 8, 041041 (2018).

[58] R. Bistritzer and A. H. MacDonald, Proc. Natl. Acad. Sci. USA 108, 12233 (2011).

[59] S. Shallcross, S. Sharma, E. Kandelaki, and O. A. Pankratov, Phys. Rev. B 81, 165105 (2010).

[60] P. Moon and M. Koshino, Phys. Rev. B 85, 195458 (2012).

[61] M. Koshino, N. F. Q. Yuan, T. Koretsune, M. Ochi, K. Kuroki, and L. Fu, Phys. Rev. X 8, 031087 (2018).

[62] H. C. Po, L. Zou, A. Vishwanath, and T. Senthil, Phys. Rev. X 8, 031089 (2018)

[63] Y.-Z. Chou, F. Wu, and S. D. Sarma, arXiv:2004.15022.

[64] P. Novelli, I. Torre, F. H. L. Koppens, F. Taddei, and M. Polini, arXiv:2005.09529.

[65] G. Li, A. Luican, J. M. B. Lopes dos Santos, A. H. Castro Neto, A. Reina, J. Kong, and E. Y. Andrei, Nat. Phys. 6, 109 (2010).

[66] G. E. Topp, G. Jotzu, J. W. McIver, L. Xian, A. Rubio, and M. A. Sentef, Phys. Rev. Res. 1, 23031 (2019).

[67] O. Katz, G. Refael, and N. H. Lindner, arXiv:1910.13510.

[68] M. Vogl, M. Rodriguez-Vega, and G. A. Fiete, Phys. Rev. B 101, 241408 (2020)

[69] M. Vogl, M. Rodriguez-Vega, and G. A. Fiete, Phys. Rev. B 101, 235411 (2020).

[70] Y. Gao, Y. Zhang, and D. Xiao, Phys. Rev. Lett. 124, 077401 (2020).

[71] M. Otteneder, S. Hubmann, X. Lu, D. Kozlov, L. E. Golub, K. Watanabe, T. Taniguchi, D. K. Efetov, and S. D. Ganichev, arXiv:2006.08324.

[72] P. Moon and M. Koshino, Phys. Rev. B 87, 205404 (2013).
[73] See Supplemental Material at http://link.aps.org/supplemental/ 10.1103/PhysRevResearch.2.032015 for technical details in numerics and derivations of the harmonic selection rules.

[74] P. Kumar, T. M. Herath, V. Apalkov, and M. I. Stockman, arXiv:2004.09732.

[75] L. M. Malard, M. H. D. Guimarães, D. L. Mafra, M. S. C. Mazzoni, and A. Jorio, Phys. Rev. B 79, 125426 (2009).

[76] S. J. Brun and T. G. Pedersen, Phys. Rev. B 91, 205405 (2015).

[77] S. Candussio, M. V. Durnev, S. A. Tarasenko, J. Yin, J. Keil, Y. Yang, S. K. Son, A. Mishchenko, H. Plank, V. V. Bel'kov, S. Slizovskiy, V. Fal'ko, and S. D. Ganichev, arXiv:2005.01407.

[78] O. E. Alon, V. Averbukh, and N. Moiseyev, Phys. Rev. Lett. 80, 3743 (1998).

[79] O. Neufeld, D. Podolsky, and O. Cohen, Nat. Commun. 10, 405 (2019).

[80] L. Zou, H. C. Po, A. Vishwanath, and T. Senthil, Phys. Rev. B 98, 085435 (2018).

[81] M. Angeli, D. Mandelli, A. Valli, A. Amaricci, M. Capone, E. Tosatti, and M. Fabrizio, Phys. Rev. B 98, 235137 (2018).

[82] S. J. Ahn, P. Moon, T.-H. Kim, H.-W. Kim, H.-C. Shin, E. H Kim, H. W. Cha, S.-J. Kahng, P. Kim, M. Koshino, Y.-W. Son, C.-W. Yang, and J. R. Ahn, Science (NY) 361, 782 (2018).

[83] W. Yao, E. Wang, C. Bao, Y. Zhang, K. Zhang, K. Bao, C. K. Chan, C. Chen, J. Avila, M. C. Asensio, J. Zhu, and S. Zhou, Proc. Natl. Acad. Sci. USA 115, 6928 (2018).

[84] T. Suzuki, T. Iimori, S. J. Ahn, Y. Zhao, M. Watanabe, J. Xu, M. Fujisawa, T. Kanai, N. Ishii, J. Itatani, K. Suwa, H. Fukidome, S. Tanaka, J. R. Ahn, K. Okazaki, S. Shin, F. Komori, and I. Matsuda, ACS Nano 13, 11981 (2019).

[85] D. Golde, M. Kira, T. Meier, and S. W. Koch, Phys. Status Solidi B 248, 863 (2011).

[86] G. Orlando, C.-M. Wang, T.-S. Ho, and S.-I. Chu, J. Opt. Soc. Am. B 35, 680 (2018).

[87] K. Chinzei and T. N. Ikeda, Phys. Rev. Research 2, 013033 (2020).

[88] K. Yao, N. R. Finney, J. Zhang, S. L. Moore, L. Xian, N. Tancogne-Dejean, F. Liu, J. Ardelean, X. Xu, D. Halbertal, K. Watanabe, T. Taniguchi, H. Ochoa, A. Asenjo-Garcia, X. Zhu, D. N. Basov, A. Rubio, C. R. Dean, J. Hone, and P. J. Schuck, arXiv:2006.13802. 